\title{
Effectiveness of External Noise Jamming in Missile Borne Monopulse Radar Receivers
}

\author{
Hari Krishna Paik, N. N. Sastry, and I. Santi Prabha
}

\begin{abstract}
This paper describes the external noise jamming of monopulse radar receiver when White Gaussian Noise (WGN) and Phase Noise (PN) signals are injected into the receiver. Initially, it is assumed that the receiver is locked onto the desired radar echo signal frequency in the presence of external noise signal as the noise power is too less to break the frequency lock of the receiver. It is shown that the Gaussian noise power required for jamming the receiver depends upon how the power is interpreted. In this paper, the Gaussian noise power is interpreted in symbol rate bandwidth, sampling frequency bandwidth, in single-sided and double-sided power spectral density. In the case of phase noise jamming, the phase noise mask $(\mathrm{dBc} / \mathrm{Hz})$ required for break-lock in the receiver is studied. It is verified that phase noise power required for jamming the receiver is less when frequency offset from the radar echo signal is large. The simulation result shows that phase noise mask of -72 is required when the frequency offset from the echo signal is $10 \mathrm{MHz}$. The effectiveness of external noise jamming is carried out through computer simulation using AWR (Visual System Simulator) software.
\end{abstract}

Index Terms-Gaussian noise, monopulse receiver, noise jamming, phase noise, power spectral density.

\section{INTRODUCTION}

Missile borne monopulse radar receivers employing phase locked loop (PLL) are mainly used to determine the positional resolution of the targets by using relative amplitude of the radar echo and interference signal [1]. These radar receivers equipped with PLL frequency tracking and servo control angle tracking subsystems are quite difficult to jam. Jamming with noise sources and repeat jammers has been successful to some extent. On-board noise jamming is possible in the specific case of mismatched monopulse sum and difference channels [2]. The receiver is said to be tracking a target perfectly if it tracks in all the three domains namely frequency, angle and range. A receiver is said to be jammed completely if and only if all the three tracking loops cited above are broken leading to the receiver tracking away from the target, so that acquisition and tracking the set points in these domains is impossible during the mission time and the target is missed out. If jamming is effective in one domain only, there is a possibility of recovery and jamming may not be successful [3].

In earlier study, it is seen that noise jamming aims at injecting interference signal into the receiver such that the desired radar signal is completely submerged by the

Manuscript received September 12, 2013; revised November 13, 2013.

Hari Krishna Paik is with the Department of EIE in V. R. S. Engg. College, Kakinada, India (e-mail: pavan_paik2003@yahoo.co.in)

N. N. Sastry is with the R\&D Wing in V. R. S. Engg. College, Vijayawada, India

I. Santi Prabha is with the Department of ECE and also is with the Empowerment of Women \& Grievances in JNTU, Kakinada, India interference as in case of denial jamming. In principle, the optimal jamming signal has the characteristics of receiver noise; in practice this may be difficult to achieve [4]. In this paper, noise jamming of missile borne monopulse radar receiver with external White Gaussian Noise (WGN) and Phase Noise (PN) signal is analyzed. For significant effectiveness of the noise jamming, WGN is chosen ideally because of maximum entropy, or uncertainty of any random waveform for a specific average power. For our simulation, a monopulse radar receiver with third order loop is designed with a typical loop bandwidth of $1 \mathrm{MHz}$. The receiver operates on unmodulated sinusoidal radar echo signal of 10 $\mathrm{dbm}$ power. The radar echo after down converted to an intermediate frequency of $30 \mathrm{MHz}$ is injected into the receiver loop along with the WGN signal and the noise power required for break-lock in the receiver is reported. It is seen that the Gaussian noise power required for break-lock in the receiver depends upon how the power is interpreted. In this context, the noise power is interpreted in symbol rate bandwidth, sampling frequency bandwidth, and in single-sided and double-sided power spectral density which are discussed in detail in the subsequent section. In another case, phase noise is generated by passing WGN through an FIR filter. This is added to the phase of the radar echo signal to simulate the phase noise which is specified through phase noise mask consisting of frequency and $\mathrm{dBc} / \mathrm{Hz}$ values and the phase noise mask required for break-lock in the receiver is presented. It is seen that phase noise power required for break-lock depends upon how the phase noise is simulated and the frequency offset from the radar echo signal.

\section{MONOPOLES RADAR PRINCIPLE}

Monopulse radars and missile seekers generally use monopulse tracking systems. Monopulse tracking systems form an angular-error on each return pulse, there by rendering the system insensitive to amplitude fluctuations on the data. This improves the radar performance and eliminates the possibilities of amplitude modulation jamming so effective against conical scanning type radar. Further, this property makes monopulse radars effective in tracking noise jamming signals that are employed against missile seekers with home-on-jam modes [5]. The block diagram of monopulse radar receiver is shown in Fig. 1.

As shown in the Fig. 1, the monopulse radar employs two overlapping antenna patterns to obtain the angular error in one coordinate. The two overlapping antenna beams are generated with a single reflector or with a lens antenna illuminated by two adjacent feeds. The two adjacent antenna feeds are connected to the two arms of a hybrid junction. The sum and difference signals appear at the two arms of the hybrid. The sum pattern is used for transmission, while both the sum and 
difference patterns are used on reception. On reception, the outputs of the sum and difference arms are each heterodyned to an intermediate frequency and amplified as, in any superhetrodyne receiver. The transmitter is connected to the sum arm from which range information of the target is extracted. The signal received with the difference pattern provides magnitude of angle error. The output of the phase detector is an error signal whose amplitude is proportional to the angular error. The angular signal actuates a servo control system to position the antenna, and the range output from the sum channel feeds into an automatic tracking unit [6].

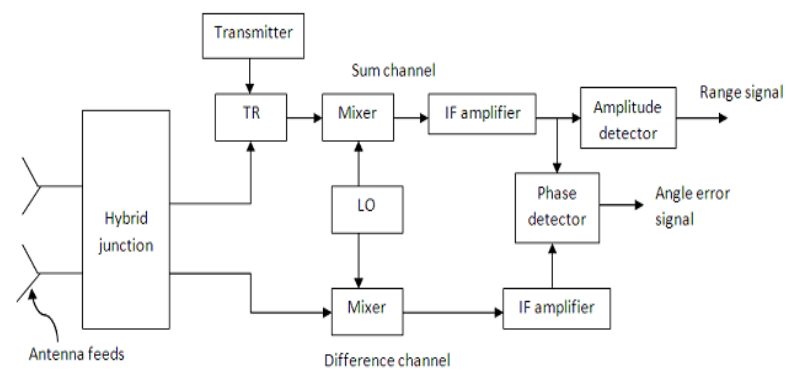

Fig. 1. Block diagram of Monopulse radar receiver

\section{RECEIVER JAMMING WITH WHITE GAUSSIAN NOISE}

The radar receiver with third order loop is shown in Fig. 2.

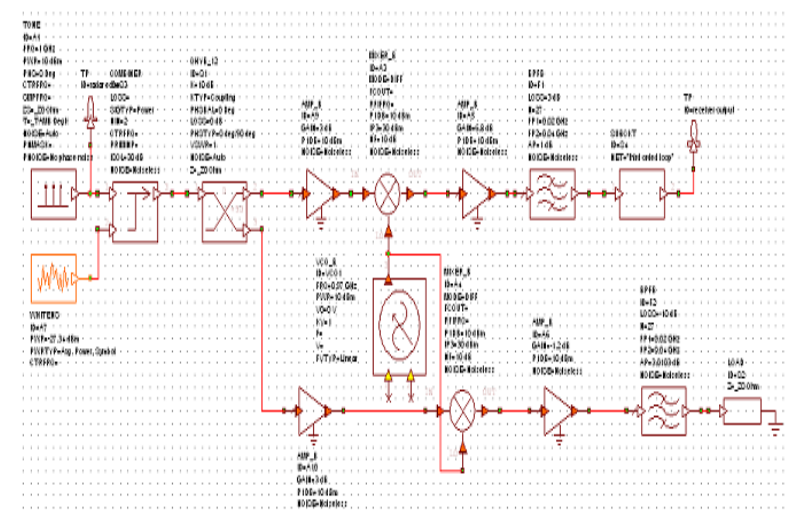

Fig. 2. Monopulse radar receiver with third order loop

With reference to Fig. 2, White Gaussian Noise (WGN) along with radar echo signal is injected into the receiver loop after down converted to an Intermediate Frequency. The WGN noise source generates independent Gaussian noise samples with zero mean. The radar echo is typically operating at $30 \mathrm{MHz}$ and at $10 \mathrm{dbm}$ power. The receiver is designed with a third order loop with a typical bandwidth of $1 \mathrm{MHz}$ using the standard method [7]. Initially, it is assumed that the receiver is locked onto the desired radar echo frequency as the Gaussian noise power is less compared to radar echo power. The Gaussian noise power is then increased without causing the loop to lose the frequency lock from the echo signal. Further, when the noise power is increased, it is seen that the loop loses the frequency lock to the radar echo signal and locks onto certain other frequency. The simulations are carried out with the Gaussian noise power estimated in symbol rate bandwidth, sampling frequency bandwidth, in single-sided and double-sided power spectral density and are discussed below.

Case-I: In the symbol rate bandwidth, the noise power is the average power in the symbol rate bandwidth. The output noise power $\left(\mathrm{N}_{0}\right)$ is specified as:

$$
P W R=\frac{N_{0}}{2} \bullet\left(\frac{f_{s}}{S M P S Y M}\right)
$$

where, $f_{s}$ is the sampling frequency and SMPSYM is samples per symbol.

Case-II: In the sampling frequency bandwidth, the noise power is the average power in the sampling frequency bandwidth. The output noise power is specified as:

$$
P W R=\frac{N_{0}}{2} \bullet f_{s}
$$

Case-III: In the single and double-sided power spectral density (PSD), the noise power is equal to the power spectral density of $\mathrm{N}_{0}$. The output noise powers for single and double sided power spectral density are specified as:

$$
\begin{array}{r}
P W R=N_{0}, \text { for single sided PSD } \\
P W R=\frac{N_{0}}{2}, \text { for doubled sided PSD }
\end{array}
$$

The entire simulation is carried out with a sampling frequency of $640 \mathrm{MHz}$ and SMPSYM of 320 with Data_rate of $2 \mathrm{MHz}$. The sampling frequency is determined as

$$
f_{s}=\text { DateRate } \bullet \text { SMPSYM }
$$

\section{A. Implementation Details}

The model generates a pseudo-random sequence of values with a Gaussian distribution using a modified version of the Box-Muller method [8]. A pair of values is generated at a time using the following:

$$
\begin{aligned}
& y_{1}=\sqrt{-2 \ln }\left(1-x_{1}\right) \bullet \sin \left(2 \pi x_{2}\right) \bullet \sigma \\
& y_{2}=\sqrt{-2 \ln }\left(1-x_{1}\right) \bullet \cos \left(2 \pi x_{2}\right) \bullet \sigma
\end{aligned}
$$

where $x_{1}, x_{2}$ are the first and second uniform deviates from the random number generator and $\mathrm{y}_{1}, \mathrm{y}_{2}$ are the two independent normal deviates with a standard deviation of $\sigma$.

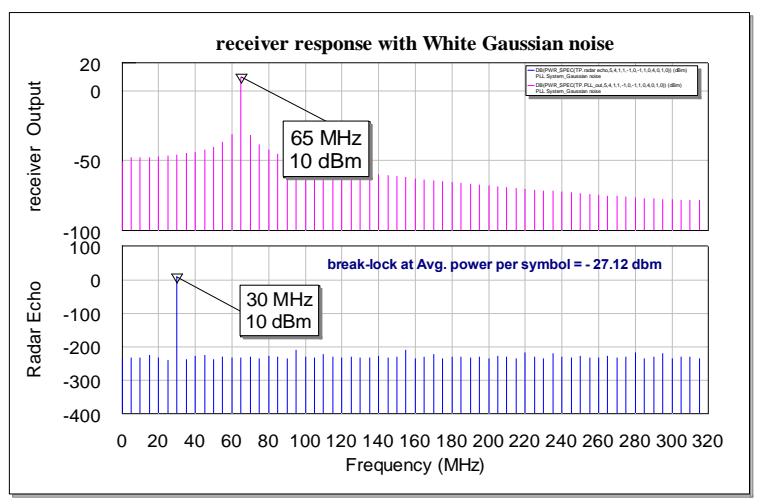

Fig. 3. (a) Receiver response in symbol rate bandwidth

The noise generated by the model has a probability density function of:

$$
p(\eta)=\frac{1}{\sqrt{\pi N_{0}}} \exp \left(\frac{-\eta^{2}}{N_{0}}\right)
$$


The receiver responses to different WGN power are shown in Fig. 3 (a), (b), (c) and (d).

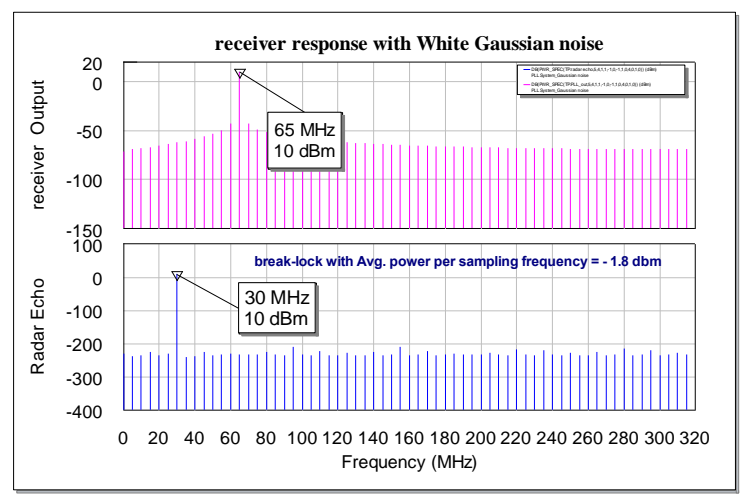

Fig. 3. (b) Receiver response in $f_{s}$ bandwidth

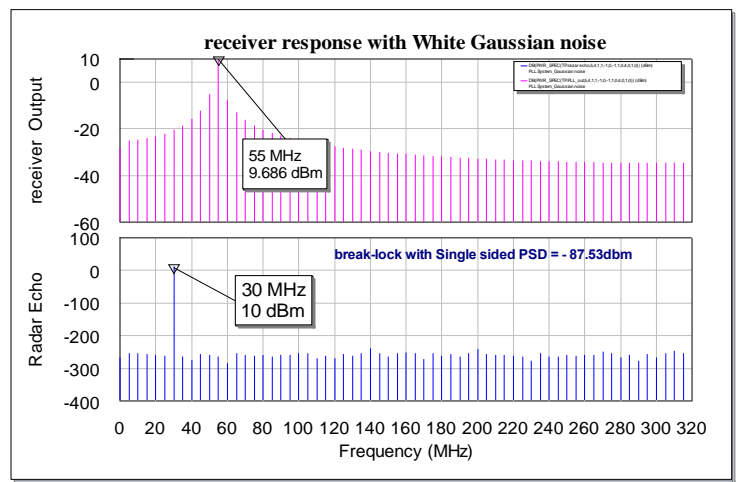

Fig. 3. (c) Receiver response in single sided PSD

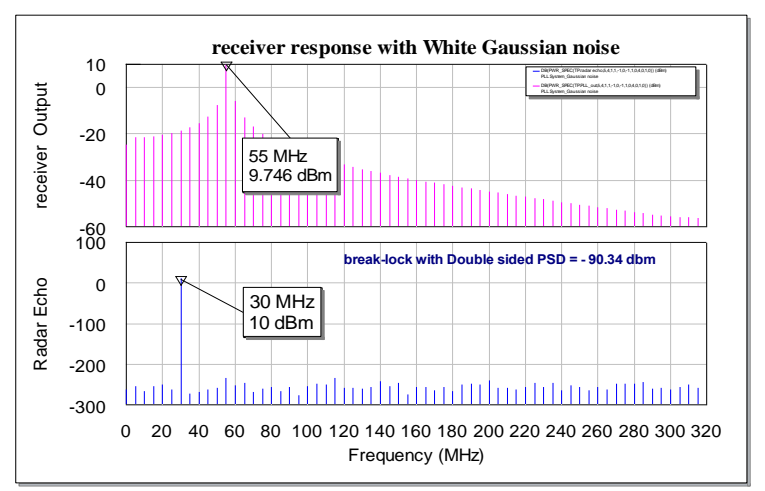

Fig. 3. (d) Receiver response in double sided PSD

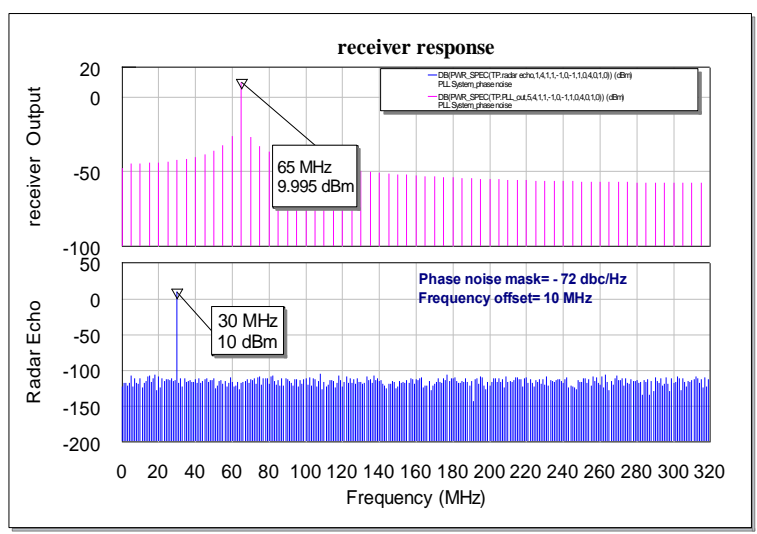

Fig. 4. (a) Receiver response to phase noise at $-72 \mathrm{dBc} / \mathrm{Hz}$

The receiver response to Gaussian noise interpreted in the symbol rate bandwidth is shown in Fig. 3. (a). It is seen that radar echo frequency is $30 \mathrm{MHz}$ and receiver output frequency is $65 \mathrm{MHz}$ which is different from radar echo frequency at Gaussian noise power of $-27.12 \mathrm{dbm}$. This shows that $-27.12 \mathrm{dbm}$ or more noise power is required to break the frequency lock of the receiver in the symbol rate bandwidth. Similarly, it is clear from Fig. 3 (b) that Gaussian noise power of $-1.8 \mathrm{dbm}$ is required to break the frequency lock of the receiver when the noise power is computed in sampling frequency bandwidth. The receiver response to the noise power in single-sided and double-sided PSD is shown in Fig. 3 (c) and (d). It is seen that when noise power is computed in double-sided PSD, the Gaussian power required for break-lock is $-90.34 \mathrm{dbm}$ and it is $-87.53 \mathrm{dbm}$ when computed in single-sided PSD. From these results it can be suggested that Gaussian power estimated in double-sided PSD should be used for effective jamming of the receiver as it is less compared to other noise power.

\section{RECEIVER JAMMING WITH PHASE NOISE}

With reference to Fig. 2, the phase noise along with radar echo signal after down converted to an intermediate frequency is applied into the receiver loop. The phase noise source generates colored noise that is added to the phase of the radar echo signal to simulate phase noise. The colored noise has non-constant amplitude spectrum. The shape of the amplitude spectrum may be specified by using frequency and $\mathrm{dBc} / \mathrm{Hz}$ values. The noise is generated by passing a white Gaussian noise signal through a FIR filter. The phase noise is specified through a phase noise mask consisting of frequency and $\mathrm{dBc} / \mathrm{Hz}$ values. The phase noise is then added to the phase of the radar echo signal and the phase noise mask required for break-lock in the receiver is observed online in the frequency spectrum of the signal.

\section{B. Implementation Details}

TABLE I: PHASE NOISE MASK REQUIRED FOR BREAK-LOCK

\begin{tabular}{|c|c|}
\hline $\begin{array}{c}\text { Prequency offset from } \\
\text { echo (MHz) }\end{array}$ & $\begin{array}{c}\text { Phase noise } \\
\text { power(dBc/Hz) }\end{array}$ \\
\hline 10 & -72 \\
\hline 12 & -75 \\
\hline 14 & -78 \\
\hline 16 & -79 \\
\hline 18 & -80 \\
\hline 20 & -81 \\
\hline
\end{tabular}

Phase noise is synthesized by passing White Gaussian noise through an FIR filter that mimics the shape of the phase noise mask. The coefficients of FIR filter are obtained from phase noise mask. The frequency specification for the filter is converted to time domain, and then a Blackmann-Harris window is applied to obtain the FIR filter coefficients. The windowing improves the general shape of the phase noise at the expense of reducing the frequency resolution. The amount of phase noise that can be successfully modeled is inversely proportional to the sampling frequency. This is due to the random phase samples wrapping around $\pm \pi$. As the amount of phase noise to be generated increases, more of the noise wraps around $\pm \pi$, effectively aliasing the noise. In general, as the average phase noise of the FIR filter bins approaches the 
inverse of the sampling frequency, the ability to generate phase noise near the desired $\mathrm{dBc} / \mathrm{Hz}$ level diminishes.

The phase noise mask required for complete break-lock in the receiver for different frequency offset from the echo signal frequency is shown in Table I.

From the Table I, it is clear that the phase noise power required for breaking the frequency lock in the receiver is less when the frequency offset from the radar echo signal is more. The receiver response to phase noise with a frequency offset of $10 \mathrm{MHz}$ and $12 \mathrm{MHz}$ are shown in Fig. 4. (a)- Fig. 4. (b).

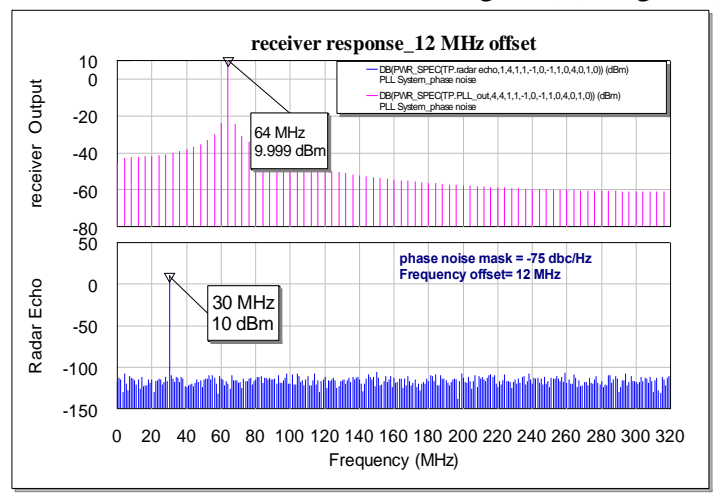

Fig. 4. (b) Receiver response to phase noise at $-75 \mathrm{dBc} / \mathrm{Hz}$

As shown in Fig. 4 (a), the radar echo signal frequency is 30 $\mathrm{MHz}$ and the receiver output frequency is $65 \mathrm{MHz}$ which is different from radar echo frequency at phase noise power of $-72 \mathrm{dBc} / \mathrm{Hz}$. So, it is clear that phase noise power of -72 $\mathrm{dBc} / \mathrm{Hz}$ is required for break-lock in the receiver with a frequency offset of $10 \mathrm{MHz}$ from the radar echo signal. Similarly, it is clear from Fig. 4 (b) that phase noise mask of $-75 \mathrm{dBc} / \mathrm{Hz}$ is sufficient to break the frequency lock in the receiver when the frequency offset from the echo signal is 12 $\mathrm{MHz}$.

\section{CONCLUSION}

Noise jamming and its effectiveness in the monopulse receiver with white Gaussian noise and phase noise signal has been carried out through extensive simulations. Results are also presented for various values of Gaussian noise and phase noise power at which break-lock in the receiver occurs. It is verified that the Gaussian noise characteristics determines the noise power required for jamming the receiver. It is shown that effectiveness of jamming the receiver is significant when Gaussian noise power is computed in double-sided PSD which is found to be $-90.34 \mathrm{dbm}$. So, from the simulation results it is suggested that Gaussian noise power computed in double-sided PSD should be preferred for effective noise jamming as the missile radar receiver can be deceived at lower power compared to other Gaussian power. In the case of phase noise jamming, the break-lock in the receiver occurs at phase noise mask of -72 and $-75 \mathrm{dBc} / \mathrm{Hz}$ when the frequency offset from the radar echo signal is 10 and $12 \mathrm{MHz}$ respectively. It is verified that the phase noise mask required

for break-lock in the receiver is less when the frequency offset from the radar echo signal is more. So, it can be recommended that phase noise with larger frequency offset is desired for effective jamming of the receiver.

\section{ACKNOWLEDGMENT}

The author would thanks to Siddhartha Academy of General \& Technical Education who provided the research facilities and technical support. We are also indebted to Director and Principal of the institute who extended support in many useful technical discussions during the progress of this work. Finally the authors wish to thanks Head of Department for his pioneering work in this area.

\section{REFERENCES}

[1] S. M. Sherman, Monopulse Principles and Techniques, Artech House, $2^{\text {nd }}$ Edition, 1984.

[2] A. F. Morabito and P. Rocca, "Optimal synthesis of sum and difference patterns with arbitrary sidelobes subject to common excitations constraints," IEEE Antennas Wireless Propag. Lett., vol. 9, pp. 623-626, 2010 .

[3] A. I. Leonov and K. I. Fomichev, Monopulse Radar, Norwood, MA Artech House, 1986.

[4] D. R. Rhodes, Introduction to Monopulse, New York: Mc Graw-Hill, 1959.

[5] D. C. Schleher, Electronic Warfare in the Information Age, Artech House, INC., 1999.

[6] D. K. Barton, Radar System Analysis and Modeling, Norwood, Artect House: MA, 2005

[7] K. O. William, An Analysis and Performance Evaluation of a Passive Filter Design Technique for Charge Pump Phased Locked Loops Application Note, National Semiconductor, 2001.

[8] S. A. Teukolsky, W. T. Vetterling, and B. P. Flannery, Numerical Recipes in C, Second Edition, pp. 289.

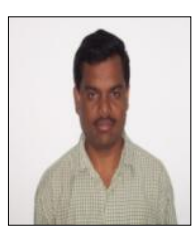

processing.

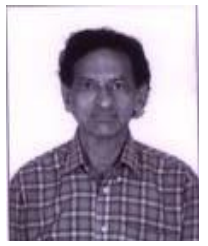

Hari Krishna Paik was born on 27 July 1975. He obtained B.E from REC, Rourkela, M.Tech from JNTU and pursuing Ph.D. (Microwave and Radar) at JNTU, Kakinada. He joined as a lecturer in the Dept. of EIE in V.R.S.Engg.College; Vijayawada in 2000. He is at present working as Assoc. Prof. in the same Dept. His area of interest is microwave and radar, digital signal Wing in V.R.S.Engg.College, Vijayawada. His area of work has been in Microwave antennas, Txs, Rxs. and EW systems. He is a member of IEEE society.

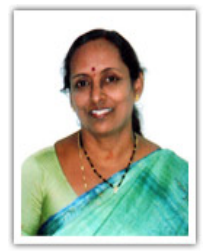

I. Santi Prabha was a professor in ECE Department and also the director of Empowerment of Women \& Grievances in JNTU, Kakinada. She did her B.Tech in electronics \& communication Engineering from JNTU college of Engineering, Kakinada and Master's degree in the area of instrumentation \& control and doctorate degree in the field of Speech Signal Processing from the same institution. She is a member of ISTE, IETE and fellow member in Institution of Engineers. 